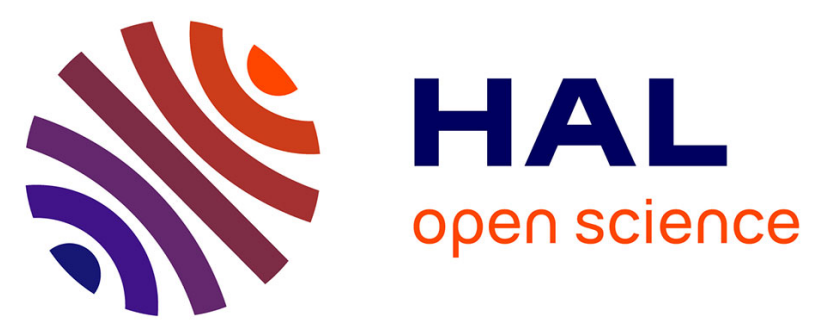

\title{
Étymologie-origine et étymologie-histoire dans le DÉRom (Dictionnaire Étymologique Roman). Coup de projecteur sur quelques trouvailles du domaine roumain Victor Celac, Eva Buchi
}

\section{- To cite this version:}

Victor Celac, Eva Buchi. Étymologie-origine et étymologie-histoire dans le DÉRom (Dictionnaire Étymologique Roman). Coup de projecteur sur quelques trouvailles du domaine roumain. Overbeck, Anja, Schweickard, Wolfgang \& Völker, Harald. Lexikon, Varietät, Philologie. Romanistische Studien Günter Holtus zum 65. Geburtstag, De Gruyter, pp.363-370, 2011. halshs-00636894

\section{HAL Id: halshs-00636894 \\ https://shs.hal.science/halshs-00636894}

Submitted on 28 Oct 2011

HAL is a multi-disciplinary open access archive for the deposit and dissemination of scientific research documents, whether they are published or not. The documents may come from teaching and research institutions in France or abroad, or from public or private research centers.
L'archive ouverte pluridisciplinaire HAL, est destinée au dépôt et à la diffusion de documents scientifiques de niveau recherche, publiés ou non, émanant des établissements d'enseignement et de recherche français ou étrangers, des laboratoires publics ou privés. 


\title{
Étymologie-origine et étymologie-histoire dans le DÉRom (Dictionnaire Étymologique Roman): coup de projecteur sur quelques trouvailles du domaine roumain
}

\author{
Victor Celac (Académie Roumaine, Bucarest) \\ \& Éva Buchi (ATILF, CNRS \& Nancy-Université)
}

\section{Introduction}

C'est lors du $25^{\mathrm{e}}$ Congrès International de Linguistique et de Philologie Romanes, en septembre 2007 à Innsbruck, que fut présenté pour la première fois le projet du Dictionnaire Étymologique Roman (DÉRom). La communication en question (Buchi/Schweickard à paraitre) se terminait par un large appel à collaboration («Romanistes de tous les pays, unissez-vous!», selon les termes du diaporama présenté), qui fut abondamment suivi par la communauté scientifique. Que le directeur de la Zeitschrift für romanische Philologie, le coéditeur du Lexikon der romanistischen Linguistik (Holtus et al. 1988-2005) et de la série des Romanistisches Kolloquium ( $c f$. en dernier lieu Dahmen et al. 2009) ait été parmi les collègues qui se sont spontanément ralliés au projet a été un sujet de grande satisfaction de la part de ses initiateurs. C'est donc avec une profonde reconnaissance que nous dédions aujourd'hui à Günter Holtus une réflexion sur le statut de l'étymologie-origine et de l'étymologie-histoire dans le dictionnaire auquel il apporte sa contribution désintéressée. ${ }^{1}$

De par son orientation résolue vers la reconstruction du protoroman (cf. Buchi/Schweickard 2008; 2009; Florescu 2009), qui privilégie une vision en quelque sorte archéologique de la recherche étymologique, le DÉRom est très clairement ancré dans ce qu'on est convenu d'appeler l'étymologie-origine. Ce choix, qui se justifie pleinement dans le cadre méthodologique de la grammaire comparée-reconstruction retenu par le projet, n'exclut pourtant pas le point de vue complémentaire de l'étymologie-histoire (cf. Baldinger 1959 et en dernier lieu le chapitre «De la etimología-origen a la etimología-historia de las palabras» de Holtus/Sánchez Miret 2008, 164-166) du champ du DÉRom. En premier lieu, cela concerne la stratification interne du protoroman, qui appartient en propre à l'étymologie panromane - et qui représente probablement l'innovation la plus importante du DÉRom. Sa caractéristique la plus saillante réside dans le fait qu'il s'agit d'une étymologie-histoire bâtie non pas, comme c'est le cas de l'étymologie-histoire idioromane, ${ }^{2}$ sur l'exploitation philologique des textes, mais entièrement sur la méthode comparative. À titre d'exemple, l'étymologie-histoire protoromane ainsi conçue a permis de mettre en évidence les stratifications suivantes dans les premiers articles du dictionnaire:

- un type originel */'థak-e-re/ et un type évolutif (syncopé) */'థ-a-re/ (Buchi 2009-2010 in DÉRom s.v. */'థak-e-/);

- un type flexionnel */'kad-e-re/, appartenant à la strate la plus ancienne du protoroman, et un type innovatif */ka'd-e-re/ (Buchi 2008-2010 in DÉRom s.v. */'kad -e-/);

- un type originel */'karpın-u/ s.f. et deux types évolutifs apparus successivement: */'karpın-u/ s.m. (changement de genre et intégration dans un paradigme majeur) et */'karpın-a/ s.f. (remorphologisation) (Medori 2008-2010 in DÉRom s.v. /'karpın-u/);

- un type originel */laks-a-/ et un type plus récent */laks-i-a-/, issu du premier par ajout de l'interfixe postradical-préflexif /-i-/ (Florescu 2010 in DÉRom s.v. /'laks-a-/);

- un type */'pont-e/ s.m. originel, un type */'pont-e/ s.f. issu de ce dernier en raison de la tendance analogique à féminiser les substantifs de la troisième déclinaison, enfin un type

\footnotetext{
${ }^{1}$ Nous remercions Marta Andronache, Jean-Paul Chauveau, Xavier Gouvert et Yan Greub (ATILF, Nancy) pour leurs notes de relecture stimulantes sur une première version de ce texte.

${ }^{2}$ Pour cette notion, cf. Buchi/Schweickard $(2009,101)$.
} 
*/'pont-e/ s.m. restauré sous la pression acrolectale (Andronache 2008-2010 in DÉRom s.v. */'pont-e/).

En raison de sa focalisation sur l'ancêtre commun des parlers romans, qui met en exergue les seules issues régulières - qu'elles aient persisté jusqu'à l'époque contemporaine ou non -, la démarche du DÉRom exclut en principe toute mention de formes déviantes. Mais en guise de concession envers ceux parmi ses lecteurs qui seraient d'abord intéressés par l'analyse des formes contemporaines, le DÉRom s'efforce de traiter minimalement, en les reléguant en note, les lexèmes irréguliers appartenant aux langues standardisées contemporaines (cf. Andronache en préparation). Dès lors, une des préoccupations des rédacteurs concerne l'établissement du rapport entre l'étymon et ces formes contemporaines, qu'elles soient dues à un accident phonétique, un changement de suffixe ou de type flexionnel ou encore à un croisement avec l'issue d'un autre étymon. C'est dans ce sens, dans la mesure où il incite à pointer du doigt les substitutions de lexèmes héréditaires réguliers par des formes secondaires, voire par des emprunts, que le DÉRom accorde une place même à l'étymologie-histoire idioromane. C'est cette facette de la question qui sera abordée dans ce qui suit.

\section{Choix de la nomenclature}

Les considérations ici exposées ${ }^{3}$ s'appuient sur six articles du DÉRom dont la rédaction a été assurée par Victor Celac et la révision finale par Éva Buchi: */a'gust-u/, */a'pril-e/,

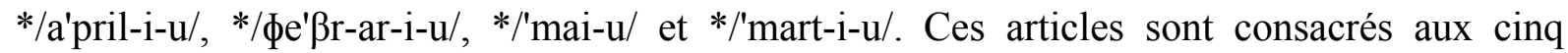
désignations de mois réputés «panromans» (Fischer 1969, 114), ce qui a motivé leur inclusion dans la nomenclature de la première phase du dictionnaire. Contrairement au focus général du DÉRom, qui se situe clairement du côté roman (et plus précisément protoroman, $c f$. Buchi à paraître), nous détaillerons ici les résultats de recherche qui se dégagent de ces six articles pour un seul idiome roman, à savoir le dacoroumain. ${ }^{4}$

Parmi ces noms de mois, mai est le seul lexème héréditaire généralisé dans toutes les variétés $\mathrm{du}$ dacoroumain. Cette situation particulière de mai semble pouvoir s'expliquer par la coïncidence formelle entre l'issue dacoroumaine héréditaire et le correspondant du slavon, slav. maj (MiklosichLexicon), qui, précisément à cause de cette coïncidence, n'a pas évincé l'issue héréditaire dacoroumaine, mais l'a revitalisée ( $c f$. déjà Mihăilă, D. 118: «lat. majus, întărit de slavon. $m a j\rangle){ }^{5}$

Les quatre autres noms de mois héréditaires en question, făurar, marţ, prier et agust, ne se sont conservés qu'en dacoroumain populaire. En dacoroumain moderne standardisé, ils ont été évincés par des emprunts au slavon (fevruarie, puis februarie; martie; aprilie; avgust, puis august), ce qui n'est pas étonnant quand on pense au caractère considérable de l'influence slavonne pendant les débuts de l'organisation sociale et de la vie religieuse des principautés roumaines et du passage à l'écrit du roumain.

\section{Désignations du mois de février}

\footnotetext{
${ }^{3}$ Les abréviations sont celles du DÉRom (cf. la liste téléchargeable sur le site http://www.atilf.fr/DERom («Consultation du dictionnaire», «Avis au lecteur»).

${ }^{4}$ Le DÉRom compte par ailleurs encore l'istroroumain, le méglénoroumain et l'aroumain parmi ses idiomes de citation obligatoire. - Pour de premiers résultats du DÉRom se dégageant pour le français, cf. Buchi et al. (à paraître); pour le portugais, cf. Benarroch (en préparation); pour le dacoroumain, cf. encore Florescu (en préparation).

${ }^{5}$ Il est possible d'admettre de plus une influence revitalisatrice postérieure de grmod. $\mu$ óïo $\left(\mathrm{cf}\right.$. Tiktin ${ }_{3}$ ).
} 


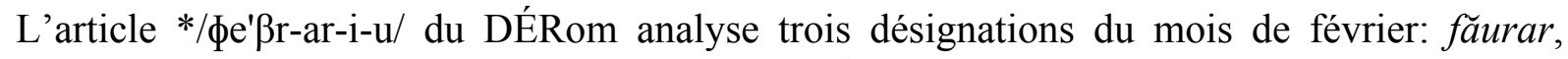
februarie et fevruarie. Les ouvrages de référence ${ }^{6}$ sont unanimes à attribuer fãurar s.m. (dp. 1622, DIR, B, 4, 91) au lexique héréditaire. Presque complètement sorti d'usage, făurar a toutefois été remplacé par fevruarie s.m. (dp. 1503, DERS), puis, notamment en dacoroumain standard, par februarie s.m. (dp. 1779, BV 2, 237). Si la plupart des ouvrages de référence sont d'accord pour considérer fevruarie comme un slavonisme $(<$ slav. fevruarij), ce à quoi nous souscrivons, la situation est plus complexe pour februarie. En effet, la majorité des ouvrages de référence (Cioranescu, $\mathrm{DEX}_{2}, \mathrm{MDA}$ ) considèrent ce lexème comme un emprunt à lat. februarius, étymologie qui se heurte notamment à un problème morphologique: $-i-u s$ (suffixe dérivationnel + suffixe flexionnel) se présente en général sous la forme -iu dans les latinismes roumains (cf. par exemple accesoriu < lat. accessorius ou comentariu < lat. commentarium). À notre avis, februarie doit être considéré comme une adaptation de fevruarie $(/ \mathrm{v} />/ \mathrm{b} /)$ sous l'influence du latin, cf. la formule de DA s.v. fevruarie: «Formă nouă, după latineşte: Februarie». Pour le dire à travers une métaphore développée lors d'une réunion de l'équipe nancéienne du DÉRom durant le post-doctorat de Victor Celac à l'ATILF (2008-2009): il ne faut pas se représenter fevruarie et februarie comme deux trains venus de deux directions différentes, mais comme un seul et même train dont un wagon $(/ \mathrm{v} />/ \mathrm{b} /)$ a été remplacé à un moment donné.

La carte ALRM SN 598 présente le lexème héréditaire făurar dans 21 points (sur un réseau de 53 points; dans les sous-dialectes de Munténie, de Transylvanie, du Maramureş et en olténien), tandis que le slavonisme originel (en /v/) n'a été relevé que dans quatre points isolés (du moldave, de l'olténien et du sous-dialecte du Banat) et les formes en /b/ dans deux points (en moldave et dans le sous-dialecte du Maramureş). Cela signifie qu'au moment des enquêtes effectuées pour cet atlas (1929-1938), le lexème héréditaire demeurait encore bien implanté dans la langue populaire, alors que le slavonisme, adapté sous l'influence du latin ou non, n'était qu'au début du processus de pénétration dans la langue populaire.

\section{Désignations du mois d'avril}

L'article */a'pril-e/ discute l'étymologie de trois lexèmes dacoroumains: aprilie, prier et priér. Tous les ouvrages de référence citent prier s.m. (dp. 1581, Coresi, Ev. 144) comme de tradition héréditaire, mais la plupart d'entre eux soit ne précisent pas l'accentuation de cette unité lexicale (EWRS; Candrea-Densusianu; Densusianu 1938, 32; Rosetti 1986, 347; Mihăescu 1993, 302), soit citent côte à côte les variantes accentuelles príer et priér sans se poser la question de la relation de filiation qui les unit, et donc sans déterminer laquelle d'entre elles est étymologique (Tiktin 3 ; DLR; MDA): la question est éludée ou considérée comme inintéressante. Seul Cioranescu semble prendre position, de façon implicite, en choisissant priér comme lemme, sans mention aucune de prier.

Or la comparaison des cognats romans (aroum. aprir, it. aprile, sard. aprile, frioul. avril, lad. auri, romanch. avrigl/avrel, fr. avril, frpr. avri, occit. abril, gasc. abriu, cat. esp. ast. gal. port. abril) permet d'établir, sans doute possible, que l'étymon protoroman est d'accentuation paroxytone (*/a'pril-e/), de sorte que c'est la forme prier, typique du dacoroumain populaire, qui se recommande comme la plus régulière.

Pour ce qui est de priér, son accentuation semble pouvoir s'expliquer par une influence analogique de noms de mois du dacoroumain populaire comme gerar 'janvier', făurar 'février', florar 'mai' ou encore gustar 'août', tous oxytons. C'est d'ailleurs cette forme priér

\footnotetext{
${ }^{6}$ Les sources documentaires sont citées sous la forme des sigles que le DÉRom leur a attribués (cf. http://www.atilf.fr/DERom, sous «Bibliographie»). Pour une réflexion générale sur la base documentaire du DÉRom, cf. Schweickard (à paraître).
} 
qui est la plus répandue en dacoroumain contemporain, ce qui lui a valu d'être retenue comme lemme par les dictionnaires de référence ( Tiktin $\left._{3} ; \mathrm{DLR}_{2} \mathrm{DEX}_{2} ; \mathrm{MDA}\right)$. Cette quasihégémonie de priér pourrait s'expliquer par un rapprochement avec le verbe a prii v.tr. 'favoriser', notamment à travers le proverbe Prier prieşte, dară şi jupeşte 'avril favorise [l'essor de la végétation], mais il peut aussi être nocif': priér aura été analysé comme un nom d'agent ('celui qui favorise') dérivé de $a$ prii.

Si la forme prier se recommande comme la plus proche de celle à laquelle on s'attend à l'issue de la triangulation entre les cognats romans et l'étymon protoroman reconstruit, elle est toutefois loin d'être régulière: d'une part, elle a subi l'aphérèse de /a/ atone, phénomène fréquent (mais pas tout à fait régulier) en dacoroumain (cf. Meyer-Lübke 1890, § 374; Densusianu 1938, 32; Rosetti 1986, 347: «lat. animalia [...] > dr. nămaie [...], lat. annotinus $[\ldots]>$ dr., ar. noatin, lat. aprilis $[\ldots]>$ dr. prier $[\ldots]$, lat. aranea $[\ldots]>\mathrm{dr}$. rîie»); d'autre part, elle présente la diphtongaison (suivie d'une diérèse) de /i/ entouré de deux /r/ (cf. Graur 1935, 50: «creier < lat. crebellum, greier < lat. grillus»); enfin, elle est marquée par la chute de /e/ final.

En tout état de cause, ces formes populaires, et d'autres encore, plus ou moins étymologiques, ont été presque entièrement évincées en dacoroumain contemporain standardisé par aprilie s.m. (dp. 1428, DERS), emprunté à slav. aprilij.

Comme pour les désignations du mois de février, la répartition dialectale est instructive, dans le sens que les zones les plus archaïques présentent le type le plus ancien. La carte ALRM SN 600 fait apparaître que le type étymologique prier est majoritaire: il est attesté dans quinze points (massivement en Munténie et en Olténie et sporadiquement en Transylvanie et dans le Banat), tandis que le type évolué priér est enregistré dans huit points (Crişana et Banat). Pour ce qui est de l'emprunt aprilie, enfin, il a été relevé dans dix points de la carte, et cela surtout en Moldavie ainsi que, plus sporadiquement, dans le Maramureş.

\section{Désignations du mois d'août}

Outre gustar (cf. ci-dessus 4.), le dacoroumain connaît quatre lexèmes désignant le mois d'août: águst, agúst, áugust et ávgust. La plupart des ouvrages de référence analysent agust s.m. (dp. ca 1650, DA), que l'on assignera au dacoroumain populaire, comme héréditaire, mais son accentuation (agúst) et donc son caractère régulier ne sont signalés explicitement que dans EWRS et DA. Et pourtant, comme dans le cas de prier/priér, l'accentuation a son importance, puisqu'on relève, à côté de l'issue régulière, une variante présentant un changement d'accent, águst, que l'on expliquera par l'influence des désignations du dacoroumain standardisé: ávgust et áugust (cf. ci-dessous). Or le rapport entre agúst et águst est loin d'avoir été saisi correctement. Ainsi $\mathrm{Tiktin}_{3}$ s.v. august enregistre plusieurs formes relevant des deux types d'accentuation, sans préciser leurs rapports de filiation. Pour ce qui est de Candrea-Densusianu et Mihăescu (1993, 302), ils donnent agust comme héréditaire, sans en préciser l'accentuation. Cioranescu, pour sa part, met la forme évoluée águst en vedette; s'il faut y voir une prise de position implicite pour le caractère étymologique de cette forme, elle doit donc être critiquée. Enfin, l'entrée manque dans MDA.

En dacoroumain moderne et contemporain standardisé, la dénomination originelle du mois d'août a été évincée par l'emprunt avgust s.m. (dp. 1593, Doc. Î. (XVI) 178). Nous suivons Ivănescu $(1980,496)$ pour considérer ce lexème comme emprunté à slav. avgustb (MiklosichLexicon), avec conservation de l'accent slave, malgré Tiktin ${ }_{1-3}$ et Cioranescu, qui proposent d'y voir un hellénisme. En effet, cette dernière hypothèse nous paraît peu probable pour des raisons historiques: le roumain a accueilli peu d'hellénismes avant la période phanariote des $17^{\mathrm{e}}$ et $18^{\mathrm{e}}$ siècles. 
Dans le cadre du mouvement relatinisant du $19^{\mathrm{e}}$ siècle, dacoroum. avgust a par la suite été remplacé par august (dp. 1881, DA). Ce dernier item de la liste des désignations dacoroumaines du mois d'août est en général (Tiktin 3 ; DA; DEX; MDA) analysé comme un emprunt à lat. augustus. Mais l'hypothèse d'un latinisme se heurte à un problème d'accentuation, puisque lat. augustus adj. 'auguste (épithète des monarques)' (TLL 2, 13911393; Ernout/Meillet ${ }_{4}$ s.v. augeō) a été emprunté sous la forme augúst adj. 'id.' (dp. 1774, DA): il n'y a pas de raison de penser que le nom aurait été emprunté avec une accentuation différente de celle de l'adjectif. À notre sens, le rapport entre avgust et august est au contraire analogue à celui qui existe entre fevruarie et februarie, tel que nous l'avons établi ci-dessus: august représente une adaptation d'après lat. augustus de avgust. En résumé, s'il nous est permis de réitérer la métaphore de tout à l'heure, august est toujours le même train que avgust, dont un seul wagon a été échangé en gare de Relatinisation.

Terminons par un coup d'œil sur la répartition dialectale des différents synonymes en lice. La carte ALRM SN 604 enregistre la forme héréditaire étymologique agúst dans treize points (surtout en Munténie et en Moldavie) et sa variante évoluée águst dans quatorze points (surtout en Transylvanie, dans le Maramureş et dans le Banat). En ce qui concerne le slavonisme, il n'a pas été relevé dans sa forme originaire (avgust), et la forme latinisée (august) n'est enregistrée que dans quatre points (en Munténie et en Moldavie). Les données dialectales se concilient donc bien avec les données historiques et les conclusions que nous en avions tirées: le type originel et héréditaire est très largement majoritaire, tandis que l'emprunt savant a peu pénétré, et surtout de façon indirecte (à travers le changement d'accent), dans ces variétés basilectales.

\section{Retour sur la lexicographie roumaine}

Les considérations qui précèdent font apparaître que, à l'exception de Candrea-Densusianu et, dans une moindre mesure, EWRS, la lexicographie historico-étymologique du dacoroumain est assez fortement orientée vers l'état actuel du lexique, en particulier dans ses choix de lemmatisation. Dans la mesure où ces dictionnaires se proposent de décrire - voire parfois de standardiser - le lexique actuel, ce trait n'a rien de critiquable en soi. Il n'empêche que, non maîtrisé, c'est-à-dire non assorti d'une gestion rigoureuse des variantes citées dans le corps de l'article, cette pratique a tendance à générer des erreurs ou du moins des insuffisances au niveau étymologique. En particulier, la lexicographie roumaine est loin de s'acquitter, même collectivement, de la tâche fondamentale de départager les formes régulières et les formes irrégulières, et encore moins de celle qui consiste à expliquer les irrégularités constatées. L'étymologie roumaine est encore essentiellement une étymologie-origine, qui se contente de mettre côte à côte un lexème contemporain et son étymon.

Il existe des exceptions à cette règle. Mais paradoxalement, tandis que les dictionnaires plus anciens (EWRS, DA, Candrea-Densusianu et Cioranescu) présentent sporadiquement des approches relevant, jusqu'à un certain point, de l'étymologie-histoire, le DLR, pourtant à la pointe du progrès sur d'autres points, pratique exclusivement l'étymologie-origine, ce qui va à l'encontre de la tendance générale en étymologie romane. En effet, les notices étymologiques de ce dictionnaire, toujours très succinctes, ne tirent aucun parti de la présentation hiérarchisée chronologiquement, pratique héritée du DA, des différents sens. Une sorte d'étymologie-histoire n'est ainsi que suggérée à travers les données philologiques, sans qu'un discours soit explicitement formulé sur les évolutions qui se dégagent de leur analyse.

Les principes de lemmatisation retenus par les auteurs des dictionnaires roumains traduit en général le souci de privilégier les formes standard ou les formes les plus répandues, ce qui constitue une stratégie lexicographique tout à fait honorable. Là où le bât blesse, c'est que les variantes qui ne bénéficient pas du statut de lemme sont presque systématiquement privées de 
traitement étymologique, de sorte que l'agencement historique de l'ensemble des formes n'est pas mis en évidence. Les irrégularités de certaines formes mises en vedette sont ainsi passées sous silence. On peut voir là le reflet d'un arrière-plan idéologique concernant la latinité «évidente» du roumain: le lexicographe peut avoir tendance à taire des irrégularités s'il les perçoit comme des déviations par rapport à une origine valorisée. Ainsi, dans la mesure où la pratique adoptée amène bien souvent à privilégier des formes non étymologiques sans que la filiation interne des variantes soit explicitée, le lecteur peut prendre par erreur une forme irrégulière pour l'aboutissement régulier de l'étymon. Dacoroum. biserică s.f. 'église' (forme actuelle standard), par exemple, ne peut pas venir directement de protorom. */ba'sil-ik-a/: protorom. */-a-/ atone aboutit à / / / Dès lors, se limiter à la mention de cet étymon est réducteur; il convient au contraire de préciser que l'aboutissement régulier en est băserică, maillon indispensable entre l'étymon et la forme contemporaine dont il conviendrait de postuler l'existence s'il n'était pas si bien attesté dans les plus anciens textes roumains. Reste ensuite à rendre compte de l'évolution /a/ $>/ \mathrm{i} /$.

En conclusion, la lexicographie roumaine bénéficierait grandement d'une appropriation de la conception moderne de l'étymologie-histoire, d'autant plus que la linguistique historique roumaine dispose tout à fait des outils, des données et des ressources humaines pour permettre un tel changement de méthode et qu'il ne sera pas difficile de trouver, au sein de la lexicographie européenne (et notamment romane) actuelle de bons modèles d'inspiration.

\section{Bibliographie}

Andronache, Marta, Le statut des langues romanes standardisées contemporaines dans le DÉRom, communication en préparation pour le $26^{\mathrm{e}}$ Congrès International de Linguistique et de Philologie Romanes (Valence, 6-11 septembre 2010).

Baldinger, Kurt, L'étymologie hier et aujourd'hui, Cahiers de l'association internationale des études françaises 11 (1959), 233-264.

Benarroch, Myriam, L'apport du DÉRom à l'étymologie portugaise, communication en préparation pour le $26^{\mathrm{e}}$ Congrès International de Linguistique et de Philologie Romanes (Valence, 6-11 septembre 2010).

Buchi, Éva, Pourquoi la linguistique romane n'est pas soluble en linguistiques idioromanes. Le témoignage du Dictionnaire Étymologique Roman (DÉRom), in: Álvarez, Xosé Afonso et al. (edd.), Actas del Simposio internacional "Por que facer Lingüística Románica no século XXI?" (Santiago de Compostela, 13-14 de novembro 2008), à paraître.

Buchi, Éva et al., Quand la linguistique française ne saurait que se faire romane: du neuf dans le traitement étymologique du lexique héréditaire, Actes du $2^{e}$ Congrès Mondial de Linguistique Française (La Nouvelle Orléans, 12-15 juillet 2010), à paraître.

Buchi, Éva/Schweickard, Wolfgang, Le Dictionnaire Étymologique Roman (DÉRom): en guise de faire-part de naissance, Lexicographica. International Annual for Lexicography 24 (2008), 351-357.

Buchi, Éva/Schweickard, Wolfgang, Romanistique et étymologie du fonds lexical héréditaire: du REW au DÉRom (Dictionnaire Étymologique Roman), in: Alén Garabato, Carmen et al. (edd.), La Romanistique dans tous ses états, Paris, L'Harmattan, 2009, 97-110.

Buchi, Éva/Schweickard, Wolfgang, À la recherche du protoroman: objectifs et méthodes du futur Dictionnaire Étymologique Roman (DÉRom), in: Iliescu, Maria et al. (éd.), Actes du $X X V^{e}$ Congrès international de linguistique et de philologie romanes (Innsbruck, 3-8 septembre 2007), à paraître.

Dahmen, Wolfgang/Holtus, Günter/Kramer, Johannes/Metzeltin, Michael/Schweickard, Wolfgang/Winkelmann, Otto (edd.), Romanische Sprachwissenschaft und Fachdidaktik. Romanistisches Kolloquium XXI, Tübingen, Narr, 2009. 
Densusianu, Ovide, Histoire de la langue roumaine. Tome II: Le seizième siècle, Paris, Leroux, 1938.

DÉRom = Buchi, Éva/Schweickard, Wolfgang (dir.), Dictionnaire Étymologique Roman (DÉRom), Nancy, ATILF, site Internet (http://www.atilf.fr/DERom), 2008-.

Fischer, Iancu, III. Lexicul. 1. Fondul panromanic, in: Rosetti, Alexandru et al., Istoria limbii române, Bucarest, Editura Academiei Republicii Socialiste România, 1969, 2, 110-116.

Florescu, Cristina, Limba română în Dictionnaire Étymologique Roman DÉRom (< Romanisches Etymologisches Wörterbuch $R E W$, in: Botoşineanu, Luminiţa et al. (edd.), Distorsionări în comunicarea lingvistică, literară şi etnofolclorică românească şi contextul european, Iaşi, ALFA/Asociaţia Culturală "A. Philippide", 2009, 153-159.

Florescu, Cristina, Éléments révélateurs du lexique roumain dans le DÉRom, communication en préparation pour le $26^{\mathrm{e}}$ Congrès International de Linguistique et de Philologie Romanes (Valence, 6-11 septembre 2010).

Graur, Alexandru, Notes sur les diphtongues en roumain, Bulletin linguistique 3 (1935), 1553.

Holtus, Günter/Metzeltin, Michael/Schmitt, Christian (edd.), Lexikon der Romanistischen Linguistik (LRL) (8 vol.), Tübingen, Niemeyer, 1988-2005.

Holtus, Günter/Sánchez Miret, Fernando: «Romanitas», Filología Románica, Romanística. Tübingen, Niemeyer, 2008.

Ivănescu, Gheorghe, Istoria limbii române, Iaşi, Junimea, 1980.

Meyer-Lübke, Wilhelm, Grammaire des langues romanes (4 vol.), Paris, Welter, 1890-1906.

Mihăescu, Haralambie, La Romanité dans le Sud-Est de l'Europe, Bucarest, Editura Academiei Române, 1993.

Rosetti, Alexandru, Istoria limbii române. De la origini şi pînă la începutul secolului al XVIIlea, Bucarest, Editura Ştiinţifică şi Enciclopedică, 1986.

Schweickard, Wolfgang, Der DÉRom (Dictionnaire Étymologique Roman) und die Arbeitsgrundlagen der romanischen etymologischen Forschung zu Beginn des 21. Jahrhunderts, Romanistik in Geschichte und Gegenwart, à paraître. 